J. Clin. Chem. Clin. Biochem.

Vol. 26, 1988, pp. 7-14

(C) 1988 Walter de Gruyter \& Co.

Berlin $\cdot$ New York

\title{
The Distribution of Unsulphated and Sulphated Glycosaminoglycans in Palmar Fascia from Patients with Dupuytren's Disease and Healthy Subjects ${ }^{1}$ )
}

\author{
By Sabine Tunn²), E. Gurr, A. Delbrück
}

Institute for Clinical Chemistry II, Center for Laboratory Medicine, Hannover Medical School, Hannover, FRG

\section{T. Buhr}

Institute for Pathology, Hannover Medical School, Hannover, FRG and

J. Flory

Department of Hand and Reconstruction Surgery, Hannover Medical School, Hannover, FRG

(Received August 24/November 11, 1987)

Summary: Eighty specimens from 20 patients with Dupuytren's disease and 7 biopsies of healthy palmar fascia were analysed for their glycosaminoglycan isomer patterns with a combined enzymatic/HPLC method. The diseased portions of palmar fascia tissue were characterized by elevated total glycosaminoglycans together with a relative increase in the sulphated fractions. The macroscopic stages of nodules, bands and unaffected tissue could be classified very well by multivariate statistical analysis on the basis of their glycosaminoglycan patterns. The biochemical analysis provided evidence of the pathological process even in those specimens that did not yet show any clinical symptoms of the disease.

\section{Introduction}

The mechanisms involved in the pathogenesis of $\mathrm{Du}$ puytren's disease are still unknown (1). Recent studies on the morphological changes in diseased tissue have demonstrated occlusion of capillaries by cells that migrate into the perivascular space. These cells are thought to be the origin of dedifferentiated cells that proliferate and metabolize in such way that they cause contracture of the aponeurosis $(2,3)$. As to the pathobiochemistry, an increase in collagen type III protein has been reported (4-7), as has an increase in the proportion of chondroitin sulphate $(4,8-11)$.

1) With support of the Deutsche Forschungsgemeinschaft (SFB 54).

2) Present address: Institute for Clinical Chemistry and Laboratory Medicine, University Hospital „Bergmannsheil“" Bochum, FRG
Because of the role played by sulphated glycosaminoglycans in the interaction with collagen (12), the question arises of whether there are changes in the proportions of glycosaminoglycans and their unsulphated and sulphated isomers in affected palmar fascia that may have an influence on the collagen pattern and the macromolecular structure. The present study was conducted to determine the types and amounts of the various sulphated and unsulphated glycosaminoglycans and to reassess the data published so far on the glycosaminoglycan content of both healthy human palmar fascia and tissue from Dupuytren's contracture, by employing a method of determination more specific than any used previously. The new method also enabled differentiation of the unsulphated, C4-sulphated and C6-sulphated components (11). 


\section{Materials and Methods}

Eighty biopsy specimens from 20 patients (aged 18 to 76 years; 2 women, 18 men) with Dupuytren's disease of the hand were taken during surgery and classified according to their macroscopic appearance as

(1) apparently normal,

(2) tissue adjacent to bands or nodules,

(3) bands, or

(4) nodules.

For reference, palmar fascia from healthy persons was taken during reconstruction surgery following accidental injury (group $0, \mathrm{n}=7$, male) and during autopsies performed within 15 hours post mortem ( $n=8$, female). The specimens were freed from adjacent tissue and blood and stored at $-20^{\circ} \mathrm{C}$ until processing. A representative sample of each specimen was set aside for microscopic examination.

The total glycosaminoglycans were isolated and the various fractions identified by specific enzymatic degradation, followed by a quantitative determination of the respective disaccharide metabolites by high performance liquid chromatography as described elsewhere (11). This method is specific and reliable, with an overall coefficient of variation of $6.8 \%$ at a substrate concentration of $100 \mathrm{nmol}$ per assay and a sensitivity of $<10$ nmol per assay. Uronic acid was determined with the method described by Bitter \& Muir (13), hydroxyproline with that of Stegemann (14) and DNA with that of Burton (15).

The microscopic examination of the specimens was performed on samples taken from the tissue portions that were analysed biochemically. Samples were stained with hematoxylin and by the van Gieson technique. The classification was made according to Millesi (16):

Stage $1 \mathrm{~A}=$ thickening and stretching of fibres,

Stage $1 \mathrm{~B}=$ development of fibre bands,

Stage 2 = cell proliferation, and

Stage 3 = few cells only, unoriented thick fibre, bundles (fig. 1).

A discriminant analysis according to Schneider (17) was performed with the SPSS-X program "Discriminant"-Release 2-.

\section{Results}

The results of the glycosaminoglycan determinations on the normal palmar fascia tissue and the four groups of diseased tissue are summarized in table 1 and figures 2 and 3 . In the most advanced stage of the disease the glycosaminoglycan content was double that found in the unaffected palmar fascia. In the early phases of the disease the hyaluronate content remained almost constant, with a decrease in the later stages, whereas the chondroitin sulphate and the dermatan sulphate fractions increased with the severity of the disease, being 10 times greater in nodules than in healthy fascia. It should be noted that the autopsy

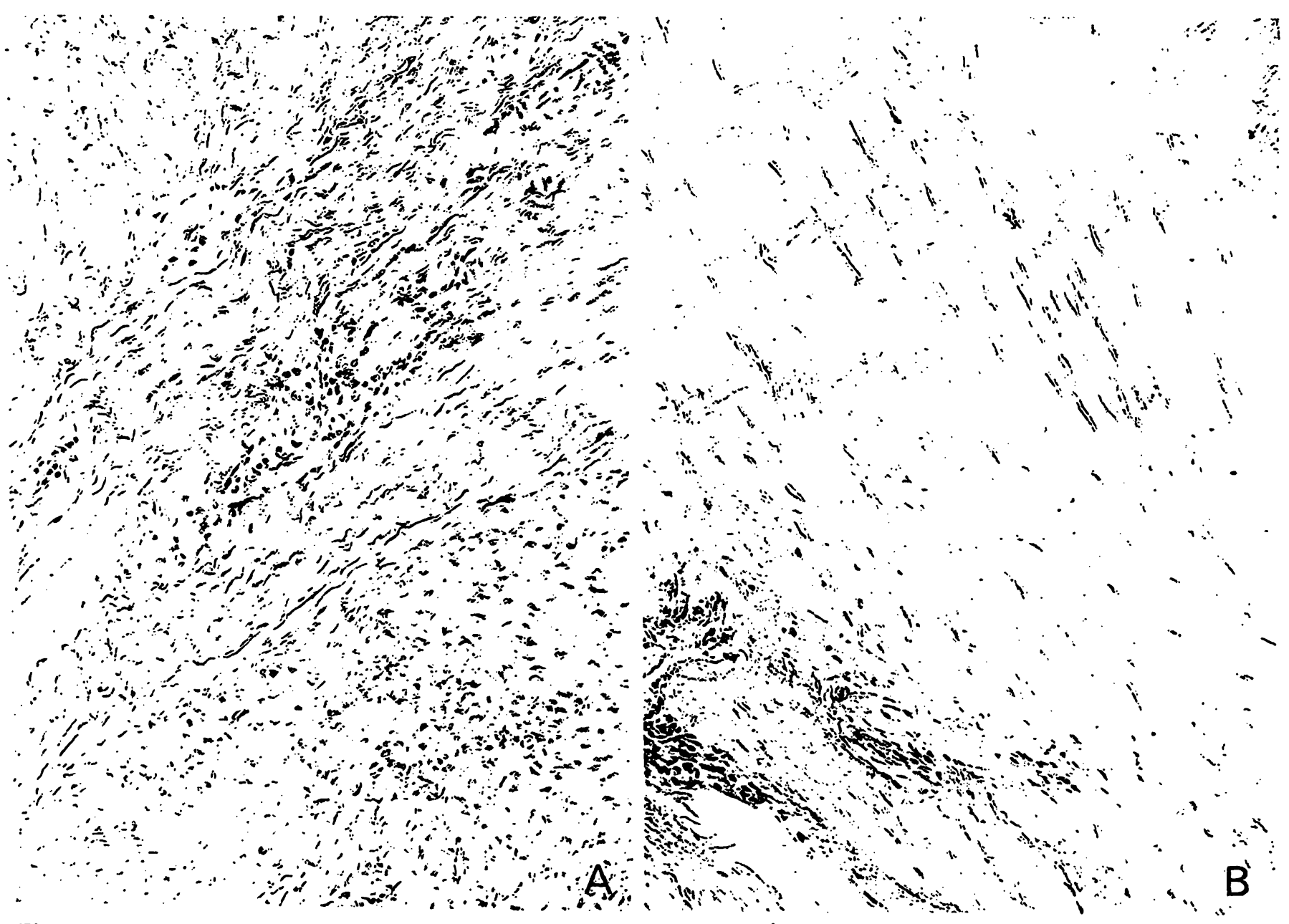

Fig. 1. Dupuytren's disease according to Millesi (16). The examples illustrate the proliferating stage 2 (A) and the' stage 3 (B, few cells only, unoriented thick fibre bundles). HE, 10x. 


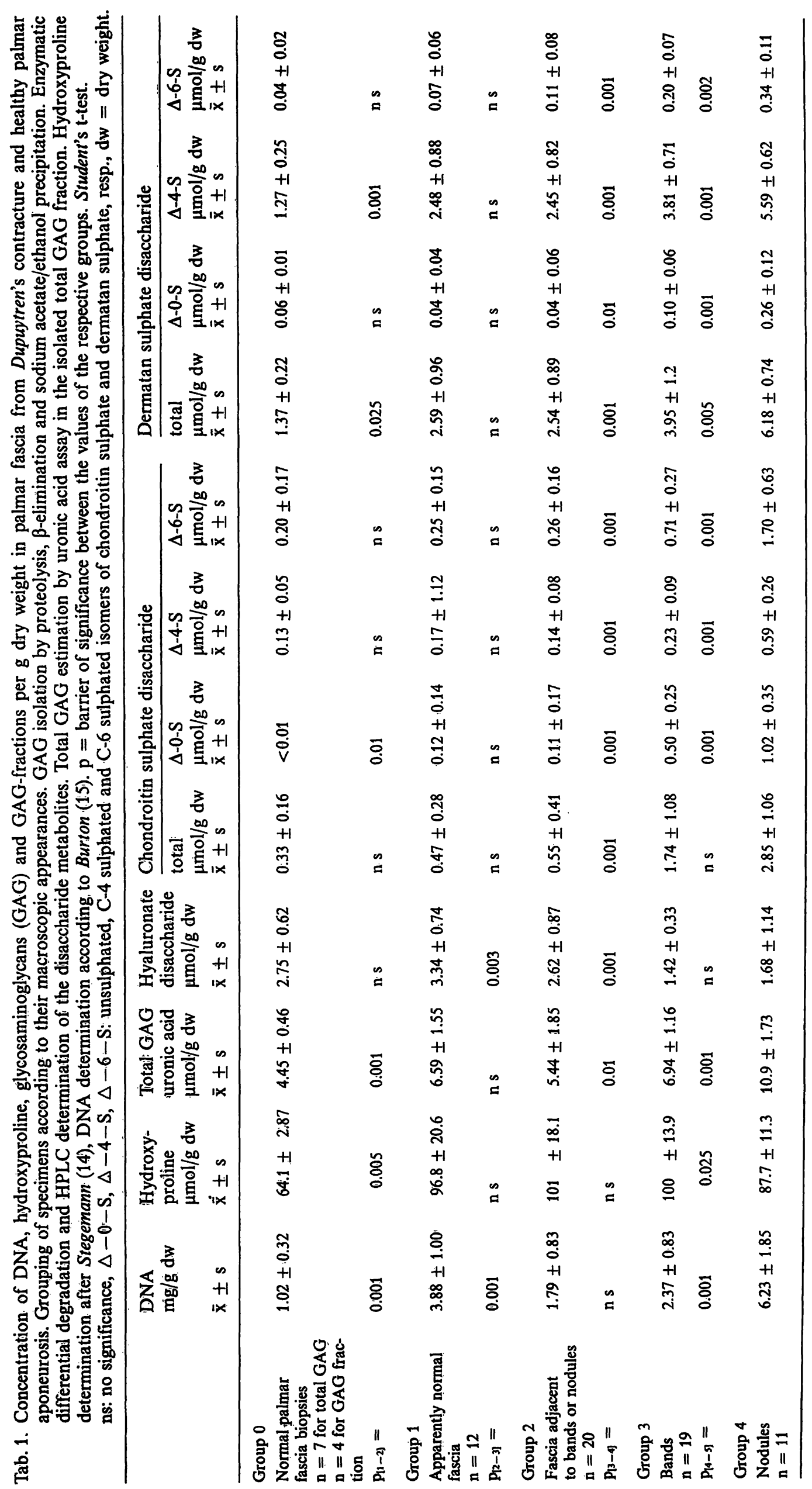




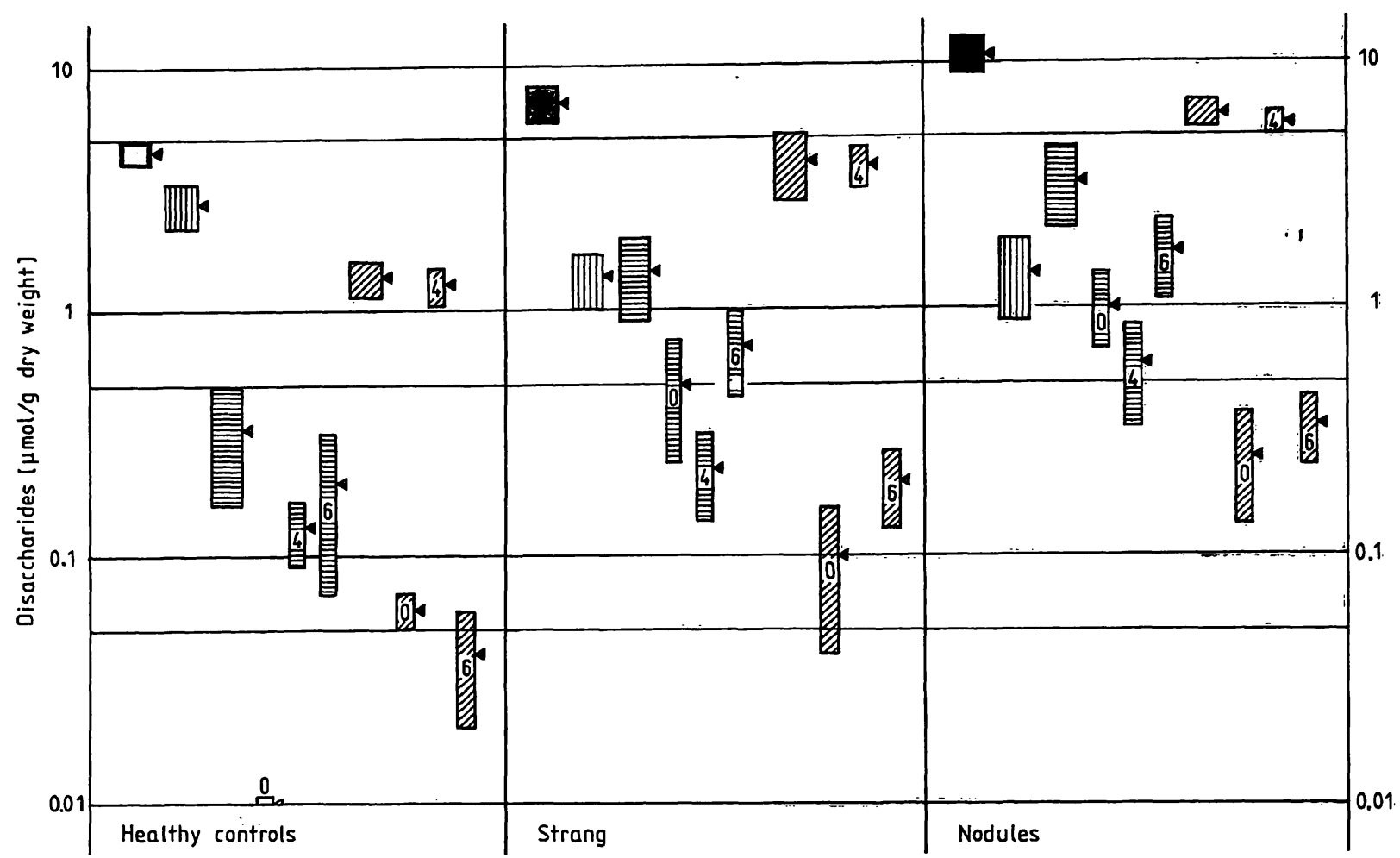

Fig. 2. Glycosaminoglycan patterns of healthy palmar fascia and in specimens from Dupuytren's disease (bands, nodules). Combined specific-enzymatic-HPLC analysis.

$\square$ glycosaminoglycan content

hyaluronate content

total condroitin sulphate fraction

国得闻 unsulphated, C4- and C6-sulphated isomers
खั⿴囗十 total dermatan sulphate fraction

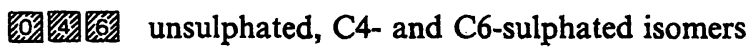

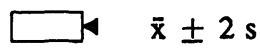

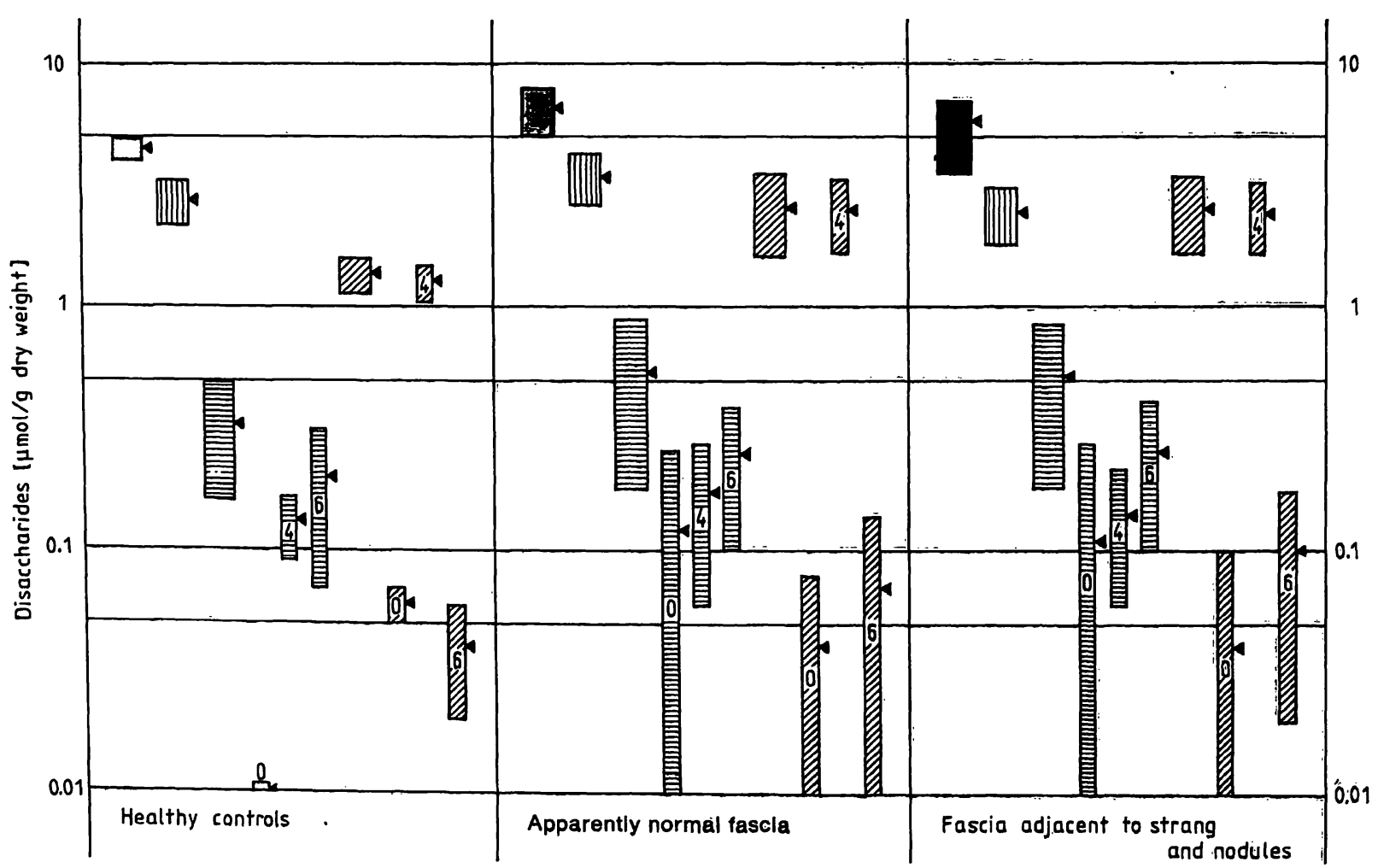

Fig. 3. Glycosaminoglycan patterns of healthy palmar fascia and in specimens from Dupuytren's disease (apparently normal tissue, tissue adjacent to bands and nodules). Combined specific-enzymatic-HPLC analysis.

Explanation see figure 2. 


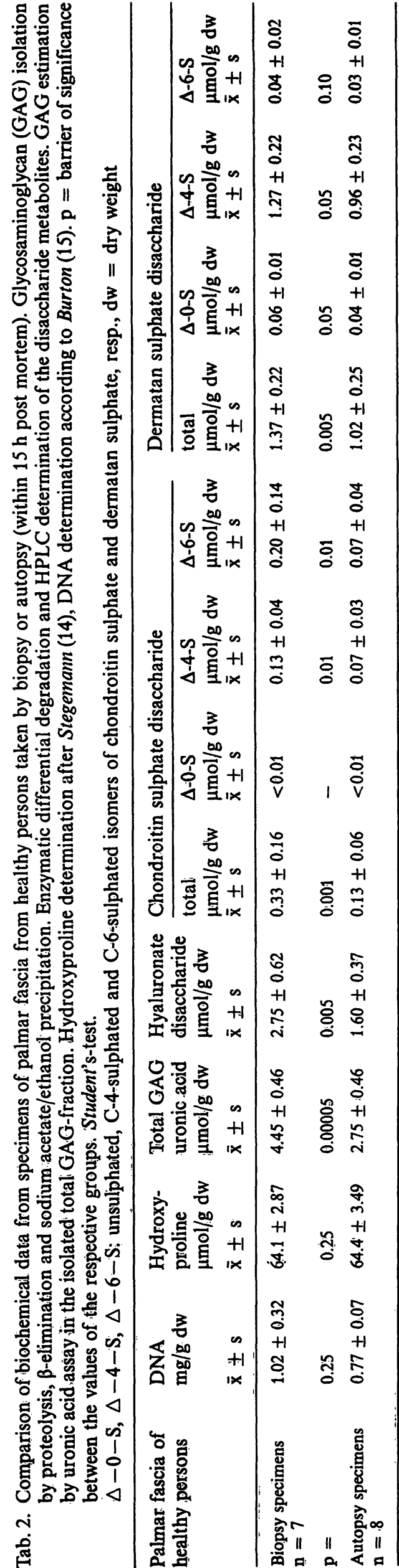

specimens of normal fascia contained smaller amounts of glycosaminoglycans than the biopsy specimens. The autopsy material proved to be unsuitable for reference because of a post mortem drop in glycosaminoglycans, mainly hyaluronate (tab. 2). However, both collagen and DNA contents remained unchanged in the autopsy specimens, compared with the biopsy material. The chondroitin sulphate isomer pattern found in Dupuytren's contracture tissue was characterized by a very marked increase in unsulphated chondroitin, which was 500 times higher than in healthy fascia (fig. 2). The main portion of the chondroitin sulphate fraction was the 6-sulphated component, which showed a concentration 10 times higher than in healthy fascia. However, in the dermatan sulphate fraction the elevation in diseased palmar fascia was due entirely to the 4-sulphated compound, which is the predominant isomer in the healthy palmar fascia (fig. 2). Regarding the unsulphated and 6-sulphated compounds, it must be kept in mind that the concentrations of these substances in palmar fascia are at the lower limit of the range of measurement of the method applied. Therefore, no conclusive statement can be made as to whether changes in the dermatan sulphate isomer pattern occur during the course of the disease (fig. 2).

Although less pronounced, the changes in the glycosaminoglycan patterns in tissue adjacent to nodules

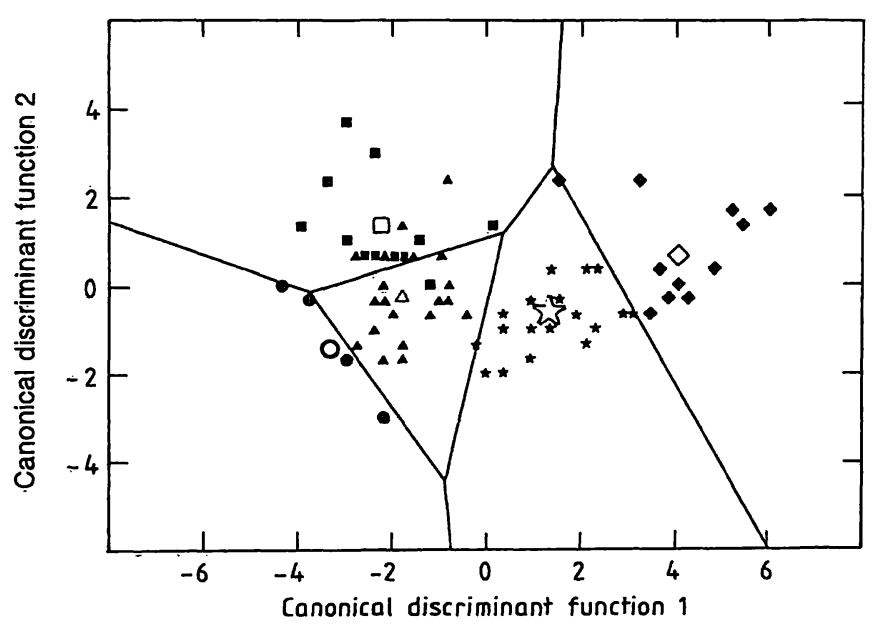

Fig. 4. Classification of specimens from healthy palmar fascia and Dupuytren's disease by multivariate statistical evaluation (18) of the glycosaminoglycan patterns: 9 variables (hyaluronate, total chondroitin sulphate, total dermatan sulphate, and the respective unsulphated, C4and C6-sulphated isomers).

- healthy control

- apparent normal fascia

$\Delta$ fascia adjacent to strang and nodules

$\star$ strang

- nodules

The open symbols indicate the respective group centroid. 
Tab. 3. Clinical, histomorphological and biochemical classification of 46 specimens from Dupuytren's disease. Clinical classification according to the macroscopic appearance of the specimen. Microscopic staging according to Millesi (16). Biochemical classification by multivariate statistical analysis (17) of the glycosaminoglycan patterns. Each number in the morphologic stage areas represents one classified specimen. Its size expresses the respective biochemical classification (stage 0: healthy palmar fascia, diseased fascia stages 1 through 4).

Microscopic staging
Clinical classification

Group 1

Apparently normal

Group 2

Tissue adjacent to bands and nodules

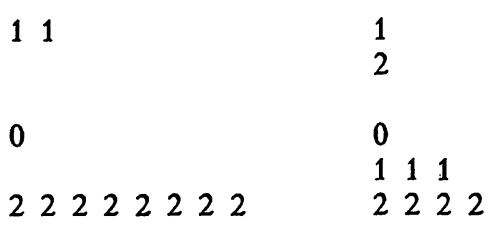

Group 3

Bands

$33333333 \quad 33333$

Group 4

Nodules

Tab. 4. Classification of results of specimens from healthy palmar fascia and Dupuytren's disease by multivariate statistical analysis (18) on the basis of 9 variables of biochemical parameters (see fig. 3). Definitions of groups predicted according to the macroscopic appearance of the specimens.

Classification of results

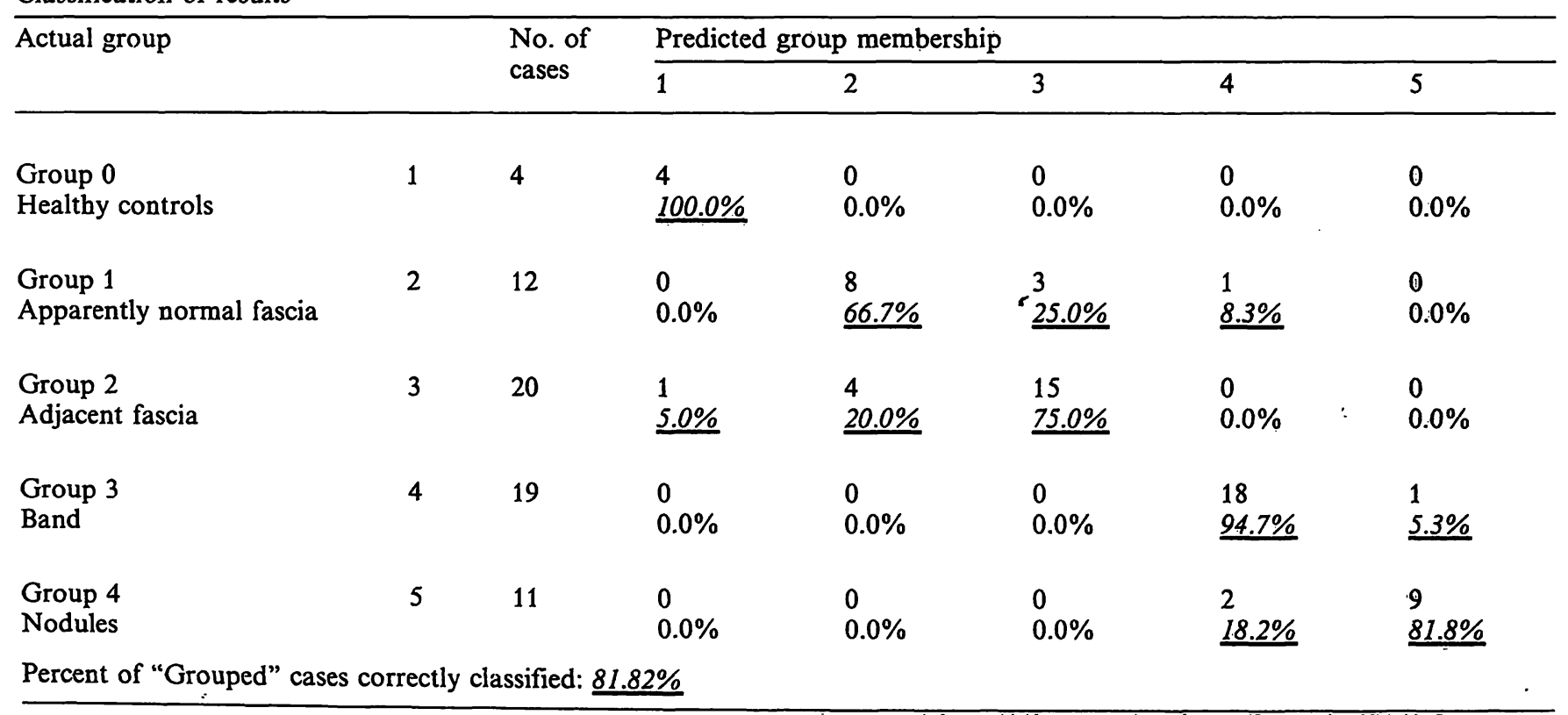

and bands as well as in apparently healthy portions of diseased palmar fascia were still great enough to classify these tissue as clearly diseased (fig. 3, tab. 1). These findings are compatible with the elevation in DNA and.in hydroxyproline concentrations (tab. 1) as well as with the increase in the number of cells (2, 18) reported for these specimens of diseased palmar fascia.
A multivariate statistical analysis (17) of the data yielded a classification that clearly distinguished between the specimens taken from nodules or bands and those from apparently normal or adjacent tissue, or from the healthy reference specimens (fig. 4, tab. 3). This biochemical classification was thus in good agreement with the macroscopic classification. A less satisfactory agreement, however, was found with the results of the microscopic examinationsi(tab. 4). 


\section{Discussion}

Data on the total glycosaminoglycan content, as well as on the distribution pattern of their fractions of palmar fascia from healthy subjects and from individuals with Dupuytren's contracture are sparse and for methodological reasons of limited value. Using electrophoresis for differentiation of the glycosaminoglycans, Flint and coworkers (8) showed that the elevation of the total glycosaminoglycans seen in $\mathrm{Du}$ puytren's tissue is based mainly on the increase in the chondroitin sulphate fraction. The findings in the present study are in general agreement with the observations of Flint et al., although no detailed comparisons can be made because the Flint findings are based on relative concentrations of glycosaminoglycans and the use of autopsy specimens for reference. In contrast, our data were obtained by specific enzymatic degradation of the glycosaminoglycans and quantitative determination of the respective disaccharide metabolites by high performance liquid chromatography. The specificity, sensitivity, and reliability of this procedure allow quantitative analyses on very small specimens from various parts of the afflicted palmar fascia and differentiation between the sulphated and unsulphated isomers of the individual glycosaminoglycans. The glycosaminoglycan isomer pattern found in the Dupuytren's contracture tissue showed an increase in the 6-sulphated compounds, and thus a change in the ratio of the 4- to the 6sulphates (fig. 2). With regard to the high synthesis rate of chondroitin-6-sulphate, it seems likely that the concentration of the unsulphated precursors should also increase. The overall ratio of unsulphated to sulphated glycosaminoglycans shifts toward the sulphated compounds because of the decrease in the hyaluronate concentration and the increase in both chondroitin and dermatan sulphate as the disease progresses. Mori \& Honda (12) presented experimental evidence for the functional importance of the proteoglycan sulphate groups in fibrillogenesis in heart valve fascia. These findings support the hypothesis that changes in the relation of sulphated to unsulphated glycosaminoglycans in Dupuytren's contracture give rise to aberrations in the structure of fibre proteins and the subsequent contracture. The biochemical changes found in the glycosaminoglycans allowed staging of the disease in which stages 3 (band) and 4 (nodules) could be clearly identified, but stages 1 (apparently normal) and 2 (adjacent tissue) could not be separated from each other or from healthy palmar fascia. However, the biochemical changes in stages 1 and 2 did provide clear evidence of the onset of the pathological process in these portions of tissue that appeared to be unaffected when examined ma- croscopically. The same was true for the microscopic examination of both stage 1 and stage 2 specimens, although the staging of a given specimen was sometimes different from the macroscopic and/or biochemical classification (tab. 4). This difference resulted from the morphological inhomogeneity of the diseased tissue and the consequent effect of sample sclection on the classification. In contrast, the biochemical analysis provided a finding that was valid for the whole specimen. In clinically and macroscopically unsuspicious palmar fascia (stage 1 in the present study) Kischer \& Speer (2) and Mohr \& Vossbeck (18) demonstrated, by electron microscopy and autoradiography respectively, a proliferation of endothelial cells in capillaries, migration of these cells and perivascular accumulation. Kischer \& Speer (2) term this early stage of the disease "pre-Dupuytren", which may correlate with our biochemically defined stage 1 (apparently normal). Cell culture experiments with cell lines derived from cells isolated from Dupuytren's contracture specimens showed an abnormal capacity to synthesise sulphated glycosaminoglycans and collagen, which the authors attribute to a permanent modulation of metabolic cell characteristics, which can be propagated in cell culture (19). In addition to the dedifferentiation of palmar fascia cells or cells of other origin; continuous stimulation of the fascia cells by abnormal matrix components or exogenous substances such as growth factors may also induce and maintain metabolic malfunctions.

In conclusion, glycosaminoglycan analysis with the combined enzymatic/HPLC method used in the present study allows characterization and classification of biopsy specimens from Dupuytren's contracture. The typical changes in the isomer fractions suggest metabolic aberrations in cells of so far unknown origin and dedifferentiation. Malcomposition of the extracellular matrix carbohydrates suggests that these carbohydrates may interact with the cell surface, thereby inducing and maintaining aberrations of metabolism and proliferation of the cells. The interactions between fibre proteins and proteoglycans are probably disturbed, with subsequent development of the contracture. Regarding the latter process, a study on the role of proteoglycans in the pathogenesis of the contracture is in progress.

\section{Acknowledgement}

The authors are greatly endebted to Miss M. Arlart and Miss $U$. Pochls for their skillful technical assistance and to Dr. $M$. Török of the Institute for Biometrics, Hannover Medical School, for her support in the statistical evaluation of the data. 


\section{References}

1. McFarlane, R. L. (1983) J. Hand Surg. 8, 703-709.

2. Kischer, C. W. \& Speer, D. P. (1984) J. Hand Surg. $9 A$, $58-62$.

3. Shum, D. T. (1985) personal communication.

4. Bazin, S., Le Lous, M., Duance, V. C., Sims, T. J., Bailey, A. J., Gabbiani, G., D’Andiran, G., Pizzolato, G., Browski, A., Nicoletis, C. \& Delaunay, A. (1980) Europ. J. Clin. Invest. 10, 9-16.

5. Menzel, E. J., Piza, H., Zielinski, C., Endler, A. T., Steffen, C. \& Millesi, H. (1979) Hand 11, 243-248.

6. Gelberman, R. H., Amiel, D., Rudolph, R. M. \& Vance, R. M. (1980) J. Bone Jt. Surg. 62, 425-432.

7. Brickley-Parsons, D., Glimcher, M. J., Smith, R. J., Albin, R. \& Adams, J. P. (1981) J. Bone. Jt. Surg. 63A, 787-797.

8. Flint, M. H., Gillard, G. C. \& Reilly, H. C. (1982) Conn. Tiss. Res. 9, 173-179.

9. Carr, T. L. (1970) Hand 1, 50-55.

10. Viljanto, J., Seppälä, P. O. \& Lehtonen, A. (1971) Ann. Rheum. Dis. 30, 423-427.

11. Gurr, E., Pallasch, G., Tunn, S., Tamm, C. \& Delbrück, A. (1985) J. Clin. Chem. Clin. Biochem. 23, 77-87.
12. Mori, Y. \& Honda, A. (1982) In: Glycosaminoglycans and Proteoglycans in Physiological and Pathological Processes of Body Systems (Varma, R. S. \& Varma, R., eds.) Karger Verlag, Basel, pp. 187-198.

13. Bitter, A. \& Muir, H. (1962) Anal. Biochem. 4, 330-334.

14. Stegemann, H. (1958) Hoppe-Seyler's Ż Physiol. Chem. $311,41-45$.

15. Burton, K. (1956) Biochem. J. 62, 315-323.

16. Millesi, H. (1981) In: Handchirurgie, Vol. 1 (Nigst, H., Buck-Gramko, D. \& Millesi, H., eds.) Georg Thieme Verlag Stuttgart-New York, pp. 15.0-15.57.

17. Schneider, B. (1970) Mathematische Grundlagen der medizinischen Diagnostik, In: Computer: Werkzeug der Medizin (Ehlers, C. Th., Hollberg, N. \& Proppe, A., eds.) Springer Verlag, Berlin, Heidelberg, New York, pp. $160-182$.

18. Mohr, W. \& Vossbeck, G. (1985) Z. Rheumatol. 44, 226230.

19. Delbrück, A. \& Schrödẹ, H. (1983) J. Clin. Chem. Clin. Biochem. 21, 11-17.

Prof. Dr. med. A. Delbrück Institut für Klinische Chemie II der Medizinischen Hochschule Hannover Krankenhaus Oststadt

Podbielskistraße 380

D-3000 Hannover 